\title{
Evolución de la leptospirosis según el Sistema de Vigilancia Epidemiológica Nacional, Chile 2003-2009
}

\author{
Paulina Martínez, Daniela Ortega y Karen Salinas
}

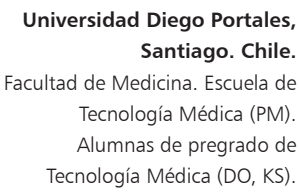

Universidad Diego Portales Santiago. Chile. Facultad de Medicina. Escuela de Tecnología Médica (PM). Alumnas de pregrado de Tecnología Médica (DO, KS)

Financiamiento: el estudio se realizó sin aporte económico o becas.

Conflicto de intereses: no existe ningún tipo de relación financiera

o personal con personas u organizaciones que pudiesen da lugar a conflicto de interés con este artículo.

Recibido: 20 de septiembre de 2011

Aceptado: 7 de octubre de 2012

Correspondencia a:

Paulina Martínez Gallegos paulinamartinezg@yahoo.com

\section{Evolution of leptospirosis according to the National Epidemiological Surveillance System, Chile 2003-2009}

Introduction: Since 2002, leptospirosis is a disease, which requires universal and immediate notification in Chile. Furthermore, it is an agent of laboratory surveillance. Aim: To describe leptospirosis in Chile and its evolution from the beginning of the obligatory notification and surveillance to the last year of available information. Methods: A descriptive analysis of the data registered from 2003 to 2009 was conducted using different databases of cases reported to the Ministry of Health (registry of notified cases, hospital discharges, laboratory registry, and deaths). As a zoonosis, animal cases registered by the Servicio Agrícola y Ganadero were also included, in order to establish an association in terms of space and temporary distribution. In accordance with the International Classification of Diseases, 10th revision, the codes A270 (icterohaemorragic leptospirosis), A278 (other forms), and A279 (leptospirosis, not specified) were distinguished. Results: Crossing of information from the different sources revealed an underreporting of the disease, making it impossible to determine the incidence rate and defining it only as a reporting rate. Discussion: According to these data, the disease tends to decrease. There was a correlation between the regions with highest human and animal cases, corresponding to the regions of Bio Bio and Maule; comparing the years of notification, there was no temporal correlation. Our study suggests that the surveillance of this disease should be further evaluated for its consistency, comparability, validity, sensitivity, and integrity.

Key words: Leptospira spp; human leptospirosis, surveillance, hospital discharges, deaths.

Palabras clave: Leptospira spp, leptospirosis humana, vigilancia, egresos hospitalarios, defunciones.

\section{Introducción}

L a leptospirosis es una enfermedad zoonótica de distribución mundial. Es causada por la bacteria espiroqueta del orden Spirochaetales, familia Leptospiraceae, género Leptospira, que afecta a casi todos los mamíferos. La infección puede ser adquirida en forma indirecta mediante el contacto con agua o suelo contaminados o directa, asociada a la exposición a animales infectados, principalmente roedores, y sus productos, fundamentalmente orina. Tanto los animales silvestres y domésticos, como roedores, ganado y caninos, son hospederos naturales.

Las espiroquetas son bacterias aerobias y móviles, con estructura helicoidal o espiral, con sobre 200 tipos patógenos de bacterias que integran el género Leptospira y ésta difiere de otras espiroquetas por la presencia de ganchos en los extremos.

La estructura antigénica de las leptospiras es complicada, siendo definida como la unidad básica el serovar, el que está representado por una cepa de referencia de acuerdo a las similitudes y diferencias antigénicas. Cada serovar tiene una conformación antigénica característica que los separa en 25 serogrupos en base a sus similitudes antigé- nicas; sin embargo, dentro de ciertos serovares pueden encontrarse cepas con pequeñas diferencias antigénicas ${ }^{1}$.

También, pueden ser clasificadas según la especie, de acuerdo a su análisis genético, siendo inicialmente detectadas la patógena Leptospira interrogans y la saprofita Leptospira biflexa. Actualmente, se han diferenciado varias especies de Leptospira ${ }^{2}$.

Los dos sistemas de clasificación basados en los términos de serovar y especie no siempre coinciden, y cepas pertenecientes al mismo serovar pueden pertenecer a diferentes especies de Leptospira. Por lo tanto, la clasificación genética puede diferir de la clasificación serológica.

Estas espiroquetas infectan a organismos vivos mediante la penetración de mucosas, conjuntiva o piel con pérdida de continuidad. Una vez en el interior, la bacteria puede inducir una amplia variedad de síntomas y signos, desde los potencialmente graves y letales, como: meningitis, neumonitis, hepatitis, pancreatitis y nefritis ${ }^{1,3}$ hasta lo asintomático o subclínico ${ }^{4}$, como lo descrito por Ganoza y cols., 2010, donde señalan hallazgos de infección subclínica ${ }^{2}$.

Recientemente esta enfermedad ha sido reconocida como un problema importante de salud pública, dado su 
incremento tanto en morbilidad como en mortalidad. La leptospira suele producir una infección crónica en mamíferos, producto de la colonización de los túbulos renales, transformándose en la fuente principal de infección para los humanos.

Los brotes o epidemias pueden estar asociados con cambios en el comportamiento humano, contaminación del agua, cambios en la densidad de los reservorios animales, o como consecuencia de desastres naturales como son las inundaciones.

Estimaciones de la OMS y la Sociedad Internacional de Leptospirosis, señalan alrededor de 350.000 a 500.000 casos anuales en el mundo, siendo considerada principalmente una enfermedad de tipo ocupacional.

La prevalencia real en América del Sur es desconocida y los resultados varían fuertemente entre los diferentes estudios, encontrándose una prevalencia de $18,9 \%$ en áreas rurales del Estado de Yucatán, México $5 ; 28 \%$ en Iquitos, Perú $^{6}$; $48 \%$ en Brasil urbano 7 ; 77,2\% en Brasil rural ${ }^{8}$; $80,6 \%$ en Venezuela; y $67,9 \%$ en Colombia urbano ${ }^{10}$.

En Chile ${ }^{3}$ se notifican anualmente alrededor de 400 casos. Otros estudios, señalan que la leptospirosis animal varía desde 88,7 a $91,7 \%$ en bovinos, $69,9 \%$ en porcinos, $37 \%$ en perros, $24,9 \%$ en ovinos a $7,1 \%$ en equinos y $47,2 \%$ en roedores silvestres ${ }^{11}$. La seroprevalencia real en humanos es desconocida; sin embargo, algunos estudios serológicos en trabajadores de riesgo presentaron positividades mayores en personal de matadero, sector pecuario y trabajadores de arrozales ${ }^{12,13}$.

Por ser considerada una enfermedad emergente $y$, con una letalidad que puede alcanzar a $20 \%$ sin tratamiento, sumado a la necesidad inmediata de aplicar medidas de control ambiental frente a la presencia de casos, el Ministerio de Salud incorporó desde julio de 2002 esta enfermedad al Decreto $N^{\circ} 158$ de Enfermedades de Declaración Obligatoria ${ }^{14}$. Por lo expuesto, es necesario - desde el punto de la salud pública-, realizar una integración de los datos provenientes desde diferentes fuentes, de manera de contribuir a su evaluación y eventual corrección.

\section{Objetivo general}

- Caracterizar la situación epidemiológica de leptospirosis en Chile y su evolución según datos del sistema de vigilancia epidemiológica nacional, desde 2003 a 2009.

\section{Objetivos específicos}

Caracterizar la situación de:

- La notificación de casos de leptospirosis humana, durante el período 2003-2009.

- La notificación de laboratorio de Leptospira spp, durante el período 2003-2009.

- Los egresos hospitalarios por leptospirosis, durante el período 2003-2007.
- Las defunciones por leptospirosis, durante el período 2003-2007.

- La notificación de casos de leptospirosis animal, durante el período 2003-2009.

\section{Materiales y Métodos}

Estudio descriptivo. Se emplearon los datos provenientes del Sistema de Enfermedades de Notificación Obligatoria (ENO) entre 2003-2009. Del mismo modo, se recogió información de la Vigilancia de Laboratorio de Leptospira spp, desde el Instituto de Salud Pública de Chile, 2003-2009. Se ha de mencionar que si bien el sistema de vigilancia de esta patología por Decreto del Ministerio de Salud, se inició en 2002, sólo se dispone de datos de laboratorio a partir de 2003, razón por la cual se ha incorporado este año como inicial.

También se consideraron las bases de registros de defunciones y egresos hospitalarios, 2003-2007, del Ministerio de Salud de Chile, correspondiendo a 2007 el último año de registro en estas bases de datos.

Para determinar la evolución histórica de los casos notificados por ENO, egresos hospitalarios y mortalidad por leptospirosis, se distinguieron, según la Clasificación Internacional de Enfermedades (CIE 10) los códigos A270 (leptospirosis icterohemorrágica), A278 (otras formas) y A279 (leptospirosis, no especificadas).

Además, se utilizaron los datos de vigilancia animal obtenidos desde el Servicio Agrícola y Ganadero (SAG) 2003-2009. Sin embargo, se ha de considerar que los casos animales son obtenidos mediante el sistema de atención de denuncias de enfermedades animales. Estas denuncias son un componente del Sistema de Vigilancia Epidemiológica y comprenden casos sospechosos de enfermedades de notificación obligatoria, enfermedades exóticas y las principales enfermedades transmisibles de ocurrencia endémica en el país.

Métodos estadísticos: En los casos notificados por vigilancia pasiva universal y vigilancia de laboratorio, se calcularon las tasas de notificación por año y región. También mediante estadísticos descriptivos se describieron las características de las personas como sexo, edad y diagnóstico (serovar).

Para el total de egresos hospitalarios por leptospirosis se estimaron proporciones por sexo y estadísticos descriptivos, de los días hospitalizados y edad. Las tasas de egresos hospitalarios fueron calculadas empleando el número de egresos en el numerador y la población nacional INE en el denominador. Las tasas de letalidad fueron estimadas empleando el número de egresos con condición de fallecidos del total de egresos hospitalarios por leptospirosis. Las defunciones específicas fueron caracterizadas según condiciones sociodemográficas, 
como situación civil, nivel de educación, actividad laboral y ocupación.

También se caracterizaron en términos descriptivos las notificaciones de casos de leptospirosis en población animal.

Se empleó el programa estadístico SPSS 17.

\section{Resultados}

\section{Vigilancia de enfermedades de notificación obligatoria}

Durante el período 2003-2009 se han notificado a través del sistema de vigilancia epidemiológica de enfermedades obligatorias (ENO) 137 casos, de los cuales $88,3 \%$ corresponden a hombres.

El número de casos ha variado desde 27 en 2003 (74,1\% hombres) a 13 en 2009 (100\% hombres). La tasa de notificación se ha desplazado desde 0,17 casos por 100 mil habitantes en 2003 a 0,08 casos por 100 mil habitantes en 2009 (Figura 1).

La edad mediana de notificación en hombres fue 41

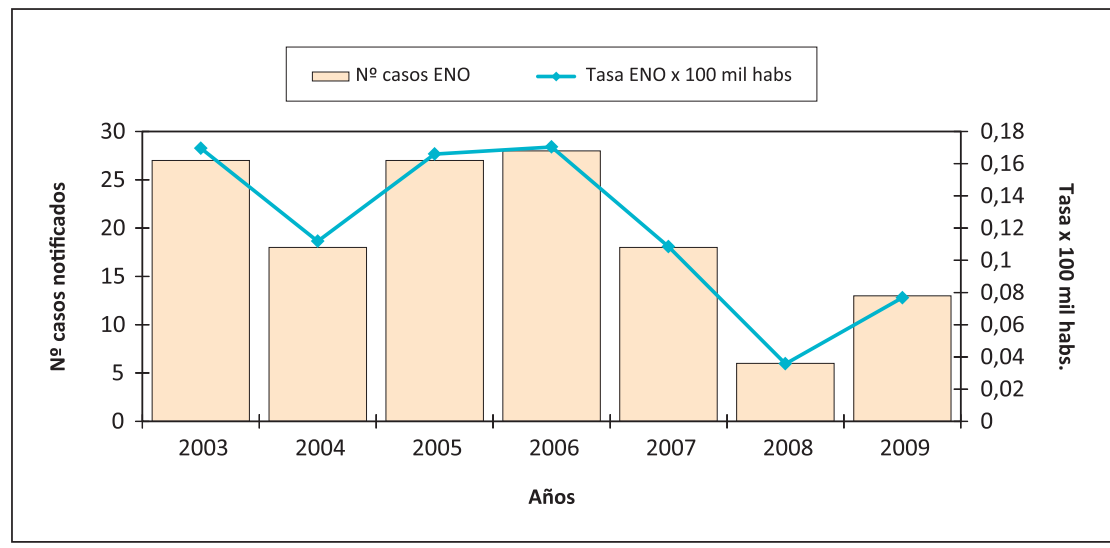

Figura 1. Número de casos y tasas de notificación acumuladas según ENO. Leptospirosis humana. Chile, 2003-2009.

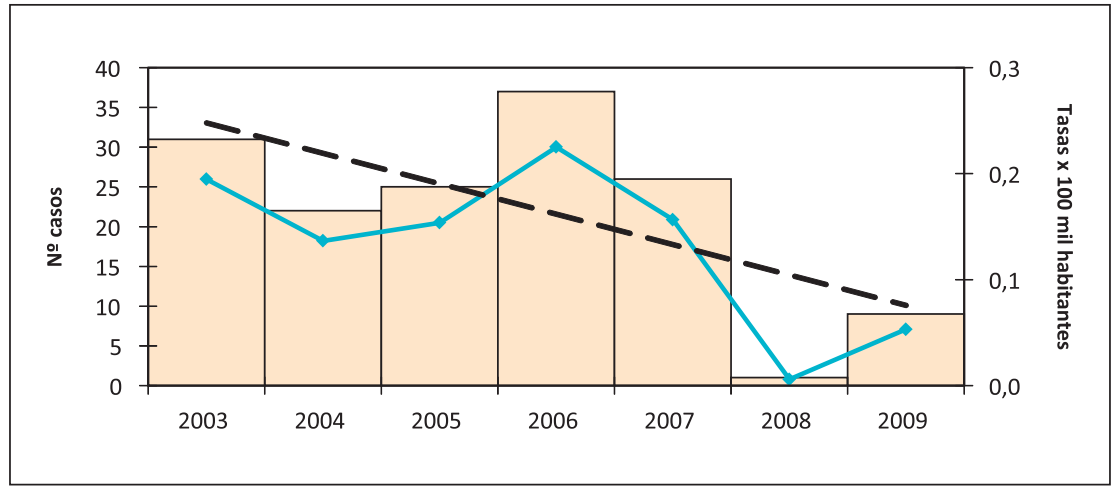

Figura 2. Casos y tasas de confirmación diagnóstica según Instituto de Salud Pública. Leptospirosis humana. Chile, 2003-2009. años (9-80 años) y en mujeres 31 años (12-66 años), existiendo diferencias estadísticamente significativas entre sexos.

Los casos acumulados según distribución geográfica se concentraron principalmente en las regiones del Maule (3,3 casos por 100 mil habitantes), Bío Bío (2,3 casos por 100 mil habitantes), Los Lagos (1,8 casos por 100 mil habitantes), Valparaíso (1,4 casos por 100 mil habitantes) y Región Metropolitana (0,3 casos por 100 mil habitantes).

\section{Vigilancia de Laboratorio}

En el diagnóstico de laboratorio se considera como prueba estándar la seroconversión mediante el incremento del título de anticuerpos por microaglutinación en tubo (MAT), la cual permite a la vez la determinación del serovar infectante.

Durante el período 2003-2009 el ISP confirmó 151 casos, concentrándose $90,7 \%$ en hombres.

El número de casos confirmados ha variado de 31 en 2003 (74,2\% hombres) a 9 en 2009 (100\% hombres), con un promedio anual cercano a 21,6 positivos. El menor y mayor número de casos confirmados se registró en 2008 (n: 1) y en 2006 (n: 37), respectivamente.

La tasa de confirmación de casos se ha mantenido en torno a un promedio de 0,13 casos positivos por $100 \mathrm{mil}$ habitantes (Figura 2).

La edad mediana de notificación fue 43 años (10-80 años); hombres 43 años (10-80 años) y mujeres 36 años (15-63 años).

Los casos acumulados según distribución geográfica se concentraron principalmente en las regiones del Maule (4,2 casos por 100 mil habitantes), Bío Bío (2,3 casos por 100 mil habitantes), Valparaíso (2,3 casos por 100 mil habitantes), Aysén ( 1 caso por 100 mil habitantes) y Región Metropolitana (0,3 casos por 100 mil habitantes).

El serovar fue determinado en $91,4 \%$ de las muestras positivas (n: 138), siendo el predominante el icterohaemorrhagiae (n: 58; 42\%), seguido por el georgia (n: 24; $17,4 \%$ ) y tercero el canicola (n: $20 ; 14,5 \%$ ).

\section{Egresos hospitalarios}

El total de egresos hospitalarios 2003-2007 corresponde a 153 pacientes $(86,3 \%$ hombres $)$.

La edad mediana fue 41 años ( $0-80$ años); en hombres fue 41,5 años (0-80 años) y en mujeres 37 años (0-79 años).

La mediana de días de estadía fue 10 días (1-80 días); en hombres 10 días (1-80 días); y en mujeres 11 días (1-16 días).

El mayor diagnóstico en egresos hospitalarios por leptospirosis, corresponde a las no especificadas o código CIE10 A279 (88,9\%).

La mayor y menor tasa/país de egresos hospitalarios fue en 2003 (0,21 egresos por 100 mil habitantes) y en 
2007 con 0,17 egresos por 100 mil habitantes, respectivamente (Figura 3).

Los egresos acumulados según distribución geográfica se concentraron principalmente en las regiones del Maule (3,5 egresos por 100 mil habitantes), Bío Bío (3,3 egresos por 100 mil habitantes), Valparaíso (1,4 egresos por 100 mil habitantes), Araucanía (0,5 egresos por 100 mil habitantes) y Los Lagos (0,5 egresos por 100 mil habitantes).

Según condición de egreso, falleció el 7,2\% de los pacientes hospitalizados. Los hombres fueron levemente más afectados que las mujeres, con una proporción de fallecidos 7,6 vs 4,8\%. La condición de fallecidos se concentró mayoritariamente en hombres en el grupo de 20-24 años (30\%) y en mujeres en el estrato de 50-54 años $(100 \%)$.

La tasa de letalidad, estimada mediante el número de fallecidos respecto al total de egresos hospitalarios por año, presentó un promedio para el período de $7,1 \%$. El porcentaje de letalidad disminuyó $43,1 \%$ entre 2003 y 2007 , presentándose $90,9 \%$ de las defunciones en hombres (10 de 11 defunciones).

\section{Mortalidad}

En el período 2003-2007 se registraron 17 defunciones por leptospirosis, concentrándose el $88,2 \%$ de ellas en hombres (n: 15).

El número de defunciones prácticamente no ha variado, 3 en 2003 (100\% hombres) a 4 en 2007 (100\% hombres), registrándose en 2005 el mayor número de defunciones (n: 6; 66,7\% hombres).

La edad mediana al fallecer en ambos sexos fue 47 años (19-69 años), en hombres 47 (19-69 años) y en mujeres 46,5 años (39-54 años).

La tasa de mortalidad se ha desplazado desde 0,019 muertes por 100 mil habitantes en 2003 a 0,024 defunciones por 100 mil habitantes en 2007 (Figura 4).

Según la distribución regional, las mayores tasas de mortalidad 2003-2007, corresponden a las regiones de Magallanes (0,64 muertes por 100 mil habitantes), Maule (0,31 defunciones por 100 mil habitantes), Bío Bío $(0,30$ decesos por 100 mil habitantes), Valparaíso ( 0,15 muertes por 100 mil habitantes), sobrepasando la tasa promedio del país $(0,10$ por 100 mil habitantes $)$.

\section{Características sociodemográficas de los fallecidos}

De los 13 fallecidos en el período 2003-2007 por leptospirosis en todo el país, 58,8\% tuvo un grado de instrucción básico o primario, mientras en hombres este nivel se incrementó a 66,7\%. En las mujeres fallecidas no hubo registro en este nivel dado que los grados de instrucción correspondieron a medio y superior ( $50 \%$ cada uno).

Sobre la condición de actividad, la mayoría al momento de la defunción se encontraba activo $(58,8 \%)$, en hombres se incrementó a $60 \%$ y en mujeres se distribuyó por partes iguales entre activas e inactivas.

De acuerdo a la categoría ocupacional, ésta se concentró mayoritariamente en el grupo de los obreros $(41,2 \%)$, aumentando en los hombres (46,7\%), mientras en mujeres no se registró defunción alguna en esta categoría.

\section{Vigilancia integrada de ENO, ISP y egresos hospitalarios}

La Tabla 1 muestra el número de casos notificados por ENO, ISP y egresos hospitalarios, donde se observa que el registro total de egresos hospitalarios es mayor que los notificados mediante ENO y los confirmados por el ISP.

El total de notificados por el ISP es 151 y los egresos hospitalarios es 153, no existiendo concordancia en el número de casos en ningún año de registro.

La diferencia entre los casos ENO y egresos hospitalarios es de 16 casos.

Al comparar el número de defunciones durante la hospitalización y el número de defunciones específicas obtenidas del registro nacional de defunciones, se observa que no existe correspondencia en 2003 y 2006, donde en

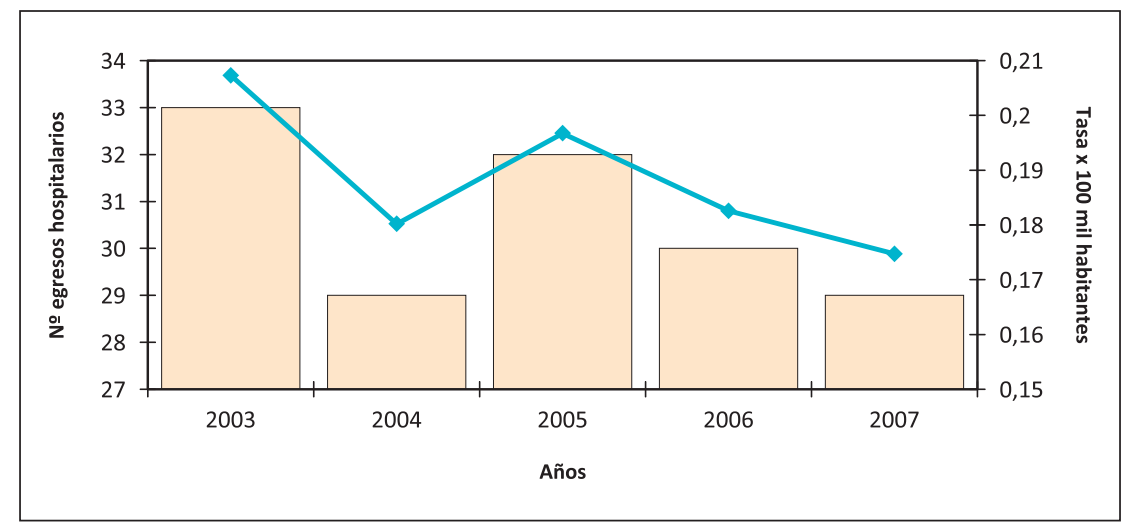

Figura 3. Número de egresos hospitalarios acumulados y tasas por leptospirosis humana. Chile, 2003-2007.

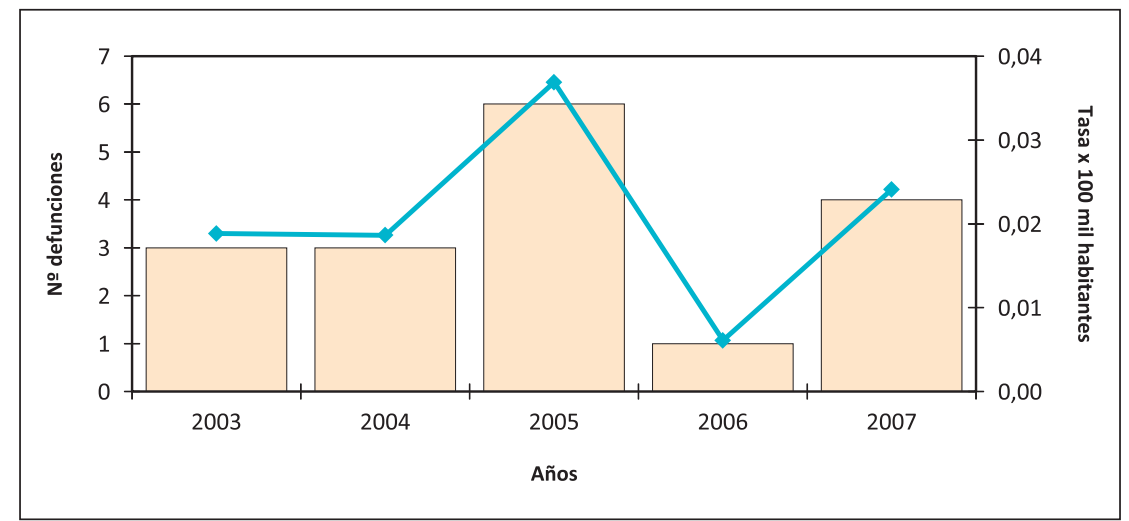

Figura 4. Número de defunciones y tasa de mortalidad acumulada. Leptospirosis. Chile, 2003-2007. 
Tabla 1. Casos de leptospirosis notificados por ENO, aislados en ISP, egresos hospitalarios* muertes hospitalarias y defunciones. Chile 2003-2009

\begin{tabular}{|cccccc|}
\hline Años & ENO & ISP & $\begin{array}{c}\text { Egresos } \\
\text { hospitalarios }\end{array}$ & $\begin{array}{c}\text { Muertes en egresos } \\
\text { hospitalarios }\end{array}$ & Defunciones \\
\hline 2003 & 27 & 31 & 33 & 4 & 3 \\
2004 & 18 & 22 & 29 & 1 & 3 \\
\hline 2005 & 27 & 25 & 32 & 2 & 6 \\
2006 & 28 & 37 & 30 & 2 & 1 \\
2007 & 18 & 26 & 29 & 2 & 4 \\
2008 & 6 & 1 & & & 17 \\
\hline 2009 & 13 & 9 & & & \\
\hline Total & 137 & 151 & 153 & & \\
\hline *2007, último año de registro. & & &
\end{tabular}

cada año el registro hospitalario presenta una muerte más que en el registro nacional de mortalidad.

\section{Vigilancia animal}

Según datos del sistema de vigilancia animal del Servicio Agrícola y Ganadero (SAG), entre los años 2003 y 2009 se notificaron 322 casos de leptospirosis, 99,4\% en bovinos y $0,6 \%$ en equinos.

En 2004 se registró el menor número de afectados (n: 9) y en 2007 se notificó el mayor número de casos (n: 134) (Tabla 2). Se registraron 22 defunciones, todas en bovinos, generando una tasa de letalidad por esta causa de $6,8 \%$.

Las regiones que concentraron mayor cantidad de casos corresponden proporcionalmente a las regiones del Maule (36,3\%), Bío Bío (23,9\%), Aysén (16,5\%), Magallanes (14\%), y Araucanía (5,6\%). Las regiones con mayores proporciones de casos animales coinciden con las mayores tasas de notificación, ya fuese por ENO como por egresos hospitalarios.

Existen regiones sin casos en animales, como las regiones de Los Ríos y Los Lagos, a pesar de ser zonas con alta población de ganado vacuno, donde al menos la región de Los Lagos sí registra casos en humanos, notificados vía ENO como egresos hospitalarios.

\section{Discusión}

Según el Decreto Supremo No 158 -desde 2002-, la leptospirosis es una enfermedad de notificación obligatoria, universal e inmediata, además de agente de vigilancia de laboratorio ${ }^{14}$. Por lo tanto, en rigor, los datos provenientes de estas tres fuentes deben coincidir. Sin embargo, no existe concordancia en el número de casos notificados por ENO, ISP y egresos hospitalarios (Tabla 1).
Tabla 2. Casos de leptospirosis en animales. Servicio Agrícola Ganadero- Chile, 2003-2009

\begin{tabular}{|cc|}
\hline Año & $\mathbf{n}$ de casos \\
2003 & 24 \\
2004 & 9 \\
2005 & 40 \\
2006 & 22 \\
2007 & 134 \\
2008 & 79 \\
2009 & 14 \\
\hline Total & 322 \\
\hline
\end{tabular}

El registro total de egresos hospitalarios es mayor que los notificados mediante ENO y los confirmados en ISP, a pesar que sólo se dispone de datos para egresos hospitalarios hasta 2007.

En la comparación del total de notificados por el ISP (151) y egresos hospitalarios (153), la diferencia es sólo de dos casos; no obstante, existe una fuerte discrepancia en la distribución de casos por año, no existiendo igualdad en ningún año de registro.

La diferencia entre los casos ENO y egresos hospitalarios es de 16 casos, a pesar de existir dos años menos de registro en los egresos hospitalarios.

Además, se sabe que no todos los casos son hospitalizados, ya que de acuerdo a lo descrito en Circular $\mathrm{N}^{\circ}$ B51/10 (febrero de 2009) ${ }^{15}$, sobre 90\% de los casos se presentan en forma leve. Por lo tanto, se podría esperar aún un número mayor de casos que los registrados en los egresos hospitalarios.

Al comparar el número de defunciones durante la hospitalización y el número de defunciones específicas obtenidas del registro nacional de defunciones, se observa que no existe correspondencia en 2003 y 2006, donde en cada año el registro hospitalario presenta una muerte más que en el registro nacional de mortalidad. Se ha de considerar que no todos los que fallecen lo hacen en un recinto hospitalario pero sí todos los que fallecen, independientes del lugar, deben ser registrados en el registro civil nacional; en consecuencia, el número de muertes en el registro de egresos hospitalarios nunca debiese ser mayor que el registro nacional de mortalidad. Por lo tanto, se observa incoherencia en el registro de defunciones en los años 2003 y 2006, donde se observa mayor número de defunciones en el registro de egresos hospitalarios que en las estadísticas vitales de mortalidad obtenidas desde los certificados de defunción, recolectados por el Registro Civil.

Tampoco existe correlación entre los casos informados 
por ENO (137) y los confirmados por laboratorio (151), a pesar que todo caso de notificación universal ENO debiese tener su confirmación de laboratorio.

En general, una forma de verificar el estado de situación de la enfermedad es mediante el cruce de datos desde las diferentes fuentes como ENO, ISP, egresos hospitalarios y defunciones, por lo tanto, en consecuencia, se desconoce el número real de casos anuales en el país.

Al realizar este análisis se observa claramente una sub-notificación de casos, verificada por un mayor número de egresos hospitalarios, a pesar que no todos los casos requieren internación en un recinto hospitalario.

En la Circular $\mathrm{N}^{\mathrm{o}} \mathrm{B} 51 / 10^{15}$ se menciona que existe una alta infección en diferentes especies animales, entre ellas: bovinos, caprinos, porcinos, ovinos, perros y ratas, lo que hace suponer que también pudiese registrarse una alta infección en humanos en las regiones que se concentran estas especies animales, lo cual es concordante con los datos encontrados en este análisis descriptivo.

Si bien, el sistema de vigilancia animal realizado por el SAG no es de carácter universal, se tiene una aproximación de la distribución temporal y geográfica. De acuerdo a ello, se evidencia concordancia entre los registros de casos humanos y la presentación de casos animales, principalmente en las regiones del Maule y Bío Bío.

Mediante el análisis de los egresos hospitalarios se confirma que los casos se concentran en hombres obreros, es decir, asociado a su condición laboral. Sí habría que preguntarse acaso los casos registrados en la Región del Maule tienen alguna relación con la producción de arroz, dado que es zona de arrozales y la literatura médica menciona mayor cantidad de casos asociados a la presencia de roedores en esta producción. Por lo demás, esta región ha notificado brotes de la enfermedad tanto en 2000 como en $2002^{16}$. También, la literatura médica ${ }^{17}$ señala que el serovar predominante en personal de arrozales es el icterohaemorrhagiae, coincidente con la mayor determinación de serovar por el ISP. Desafortunadamente, estas preguntas no pueden ser resueltas con la información disponible.

Luego del cruce de información desde las diferentes fuentes, se puede apreciar una sub-notificación de la enfermedad, imposibilitando la determinación de la tasa de incidencia de casos y definiéndola sólo como tasa de notificación. De acuerdo a estos datos, la enfermedad tiende a disminuir.
Finalmente, a ocho años del inicio del sistema de vigilancia epidemiológica nacional, se puede señalar que este sistema probablemente deba ser sujeto a una evaluación en términos de su consistencia, exactitud, comparabilidad, validez, sensibilidad e integralidad.

Agradecimientos. A Paulina Méndez R. (MV). Subdepartamento de Vigilancia Epidemiológica. División Protección Pecuaria. Servicio Agrícola y Ganadero, por su generosa participación en la entrega de datos desde el sistema de vigilancia animal.

\section{Resumen}

Introducción: En Chile, a partir de 2002 la leptospirosis es una enfermedad de notificación obligatoria, universal e inmediata, además de ser agente de vigilancia de laboratorio. Objetivo: Describir la leptospirosis en Chile y su evolución desde el inicio del sistema de vigilancia obligatoria hasta el último año de información disponible. Métodos: Se realizó un análisis descriptivo de los datos registrados desde 2003 al 2009, considerando las diferentes fuentes del Ministerio de Salud que notifican casos (registro obligatorio de casos, egresos hospitalarios, registro de laboratorio y defunciones). Por ser una zoonosis también se consideraron los casos animales registrados por el Servicio Agrícola y Ganadero, de manera de establecer una asociación en términos de su distribución espacial y temporal. Se distinguieron según la Clasificación Internacional de Enfermedades (CIE 10) los códigos A270 (Leptospirosis icterohemorrágica), A278 (Otras formas) y A279 (Leptospirosis, no especificadas). Resultados: Luego del cruce de información desde las diferentes fuentes, se puede apreciar una sub-notificación de la enfermedad, imposibilitando la determinación de la tasa de incidencia y definiéndola sólo como tasa de notificación. Discusión: De acuerdo a estos datos, la enfermedad tiende a disminuir. Existe correlación entre las regiones que registran mayor número de casos humanos y animales, correspondiendo a las regiones del Bío Bío y Maule, no existiendo concordancia en los años de registro de casos. Se sugiere que el sistema de vigilancia de esta enfermedad sea sometido a una evaluación, en términos de su consistencia, exactitud, comparabilidad, validez, sensibilidad e integralidad.

\section{Referencias bibliográficas}

1.- Organización Mundial de la Salud (OMS) Leptospirosis Humana: Guía para el Diagnóstico, Vigilancia y Control 2008; 127 p.

2.- Ganoza Ch, Matthias M, Saito M,
Céspedes M, Gotuzzo E, Vinetz J. Asymptomatic renal colonization of humans in the Peruvian amazon by leptospira. PLOS Negl Trop Dis 2010; 4 (2): e612.

3.- Abuauad C, Osorio G, Rojas J P, Pino L. Leptospirosis: Presentación de una infección fulminante y revisión de la literatura. Rev
Chilena Infectol 2005; 22 (1): 93-7.

4.- American Academy of Pediatrics. Section 3. Summaries of Infectious Diseases.

Leptospirosis. En Pickering LK, Baker CJ, Kimberlin DW, Long SS, eds. Red Book 2012 Report of the Committee on Infectious Diseases. Elk Grove Village, Il. American Academy of 
Pediatrics 2012. pp: 469-471.

5.- Zavala, Pinzón J, Flores M, Damian A G. La leptospirosis en Yucatán: Estudio serológico en humanos y animales. Salud Pública Mex 1984: 26: 254-9.

6.- Johnson M A, Smith H, Joeph P, Gilman R H, Bautista C T, Campos J K. Environmental exposure and leptospirosis, Peru. Emerg Infect Dis 2004; 20: 1016-22.

7.- Simões A, De Lourdes M. Locales probables de infección y ambiente de ocurrencia de la leptospirosis humana en el Brasil, 2001 a 2003. Memorias de II Taller Internacional y II Reunión Científica. La Habana-Cuba. 2004.

8.- De Lourdes M, Arsky S, Kleber W, Cerqueira R, Strugo A H. Probable areas of infection and ambience of occurrence of human leptospirosis in Brazil 2001-2003. Rev Cubana Med Trop 2005: 57 (1): 59-60.

9.- Vado S, Cárdenas M, Laviada M H, Vargas P F, Jiménez D B, Zavala V J. Estudio de casos clínicos e incidencia de leptospirosis humana en el Estado de Yucatan, México durante el período 1998 a 2000. Rev Biomedica 2002; 13: 157-64.

10.- Rodríguez H, Lozano C, Bedoya C, Grondona L. Prevalencia de leptospirosis en humanos en la zona urbana del municipio de Puerto Libertador, Córdoba, Colombia. Revista de Investigación Agraria y Ambiental 2009; 0 (1): 23-8.

11.- Zunino E, Pizarro R. Leptospirosis. Puesta al día. Rev Chilena Infectol 2007; 24 (3): 220-6.

12.- Luchsinger E, Zamora J. Tipificación de leptospirosis en ganado bovino. Rev Soc Med
Vet 1967; 17: 21-31.

13.- Zamora J, Riedemann S, Montecinos M I, Cabezas X. Encuesta serológica de leptospirosis humana en ocupaciones de alto riesgo en Chile. Rev Med Chile 1990; 118: 247-52.

14.- Gobierno de Chile. Ministerio de Salud. Reglamento sobre notificación de enfermedades transmisibles de declaración obligatoria $\mathrm{N}^{\mathrm{o}} 158$. Santiago, 22 de octubre de 2004.

15.- Gobierno de Chile. Ministerio de Salud. Circular de vigilancia y control de Leptospirosis. 2009.

16.- Arias H, Núñez M, Valenzuela I, Olivares A. Brote epidémico de leptospirosis en niños de Linares. Rev Chil Pediatr 2003; 74 (4): 405-10.

17.- Levett P. Leptospirosis. Clin Microbiol Rev 2001; 14 (2): 296-326. 